\title{
Integrated gold superparticles into lateral flow immunoassays for the rapid and sensitive detection of Escherichia coli 0157:H7 in milk
}

\author{
Yu Li, ${ }^{1,2 *}$ Xirui Chen, ${ }^{1,2 *}$ Jing Yuan, ${ }^{1,2}$ Yuankui Leng, ${ }^{1,2}$ Weihua Lai, ${ }^{1,2}$ Xiaolin Huang, ${ }^{1,2} \dagger$ ( \\ and Yonghua Xiong ${ }^{1,2,3}$ (i) \\ ${ }^{1}$ State Key Laboratory of Food Science and Technology, Nanchang University, Nanchang 330047, P. R. China \\ ${ }^{2}$ School of Food Science and Technology, Nanchang University, Nanchang 330047, P. R. China \\ ${ }^{3}$ Jiangxi-OAI Joint Research Institute, Nanchang University, Nanchang 330047, P. R. China
}

\begin{abstract}
Escherichia coli O157:H7 is a common harmful foodborne pathogen that can cause severe diseases at low infectious doses. Traditional lateral flow immunoassay (LFIA) for the rapid screening of E. coli O157:H7 in food suffers from low sensitivity due to its dependence on 20- to 40-nm gold nanoparticles (AuNP) with insufficient brightness as labels. To address this issue, we reported for the first time the successful synthesis of gold superparticles (GSP) by encapsulating numerous small AuNP into a polymer nanobead using an evaporation-induced self-assembly method. Results indicated that the resultant GSP exhibited remarkably enhanced absorbance compared with the most widely used $40 \mathrm{~nm}$ AuNP in LFIA. In addition, the absorbance of GSP could be easily tuned by varying GSP sizes. Under optimized conditions, we achieved a rapid and sensitive determination of E. coli $\mathrm{O} 157: \mathrm{H} 7$ in milk with a detection limit of $5.95 \times 10^{2} \mathrm{cfu} / \mathrm{mL}$ when using the GSP with a size of $342 \mathrm{~nm}$ as LFIA signal reporters, exhibiting improvement of approximately 32 -fold relative to the conventional $40 \mathrm{~nm}$ AuNP-LFIA method. We further demonstrated the selectivity, accuracy, reliability, and practicality of the proposed GSP-LFIA strip. In summary, this work offers a new strategy for improving LFIA sensitivity using assembled GSP as markers and demonstrates huge potential in rapidly and sensitively detecting foodborne pathogens.
\end{abstract}

Key words: gold superparticles, lateral flow immunoassays, Escherichia coli O157:H7, milk

\section{INTRODUCTION}

Escherichia coli O157:H7 is a common harmful foodborne pathogen that can cause infections in humans and

Received November 20, 2019.

Accepted March 23, 2020.

*These authors contributed equally to this work.

†Corresponding author: hxl19880503@163.com animals through contaminated food and water (Mead and Griffin, 1998). Previous studies have indicated that $E$. coli $\mathrm{O} 157: \mathrm{H} 7$ is associated with severe clinical symptoms, such as diarrhea, hemorrhagic colitis, and hemolytic uremic syndrome even at low infectious doses (approximately 10-100 cells; Tarr, 1995; Lin et al., 1996; Sperandio et al., 1999; Setterington and Alocilja, 2011). Traditional culture-based methods are widely regarded as the gold standard for monitoring E. coli O157:H7 contamination. However, these methods frequently suffer from several limitations. For example, they are time consuming $(\sim 7 \mathrm{~d})$ and cannot provide on-site feedback (Deisingh and Thompson, 2004). Various rapid detection methods such as PCR (Samad et al., 2018), ELISA (Guo et al., 2016), and nanomaterial-based biosensors (Sun et al., 2015) have been developed recently. However, the sensitivity of these methods typically ranges from $10^{3}$ to $10^{6} \mathrm{cfu} / \mathrm{mL}$. Therefore, the development of a sensitive detection method for on-site and rapid screening of E. coli O157:H7 remains a considerable challenge.

Lateral flow immunoassay (LFIA) has been widely used in the field detection of foodborne pathogens due to its simplicity, rapidity (15 min), specificity, and low cost (Zhang et al., 2015; Huang et al., 2016; Jiang et al., 2016; Luo et al., 2017; Huang et al., 2018a; Chen et al., 2020). However, traditional LFIA using 20- to 40$\mathrm{nm}$ gold nanoparticles (AuNP) as labels suffers from low sensitivity because of the insufficient brightness of AuNP (Jiang et al., 2018). A significant approach for improving the sensitivity of conventional AuNP-based LFIA is to increase AuNP accumulation around an analyte in the test line region of a strip and to amplify the colorimetric signal intensity of AuNP because of the enlarged collective molar extinction of AuNP (Zhou et al., 2019). To achieve this objective, antigen-antibody reaction (Choi et al., 2010), nucleic acid hybridization (Hu et al., 2013), and biotin-streptavidin (Taranova et al., 2017) mediated signal amplification systems based on the formation of dual AuNP conjugation or AuNP aggregates have been used to enlarge AuNP accumula- 
tion. However, such amplification strategies are typically completed after ending the strip test. Thus, these strategies lead to additional experimental procedures, compromising the simplicity of LFIA. Another strategy for increasing the signal intensity of AuNP on the test line is directly using cross-linked AuNP aggregates prepared via diverse cross-linking technologies such as oligonucleotide and polyamidomine dendrimers (Liu et al., 2006; Shen et al., 2013a,b; Zhu et al., 2014). However, the synthesis of these AuNP aggregates frequently suffers from considerable batch-to-batch variation. This condition largely limits their wide application. In addition, the AuNP-doped nanocarriers, including carbon nanotubes (Qiu et al., 2015), silica nanorods (Takalkar et al., 2016), and bacteria (Huang et al., 2018b), have also been used to increase AuNP accumulation for amplifying colorimetric signal intensity to enhance LFIA sensitivity (Duan et al., 2017). Nonetheless, these micrometer-sized nanocarriers may result in decreased diffusion rates and unwanted background residue on the nitrocellulose (NC) membrane, and consequently, deteriorated LFIA sensitivity. Moreover, a limited surface restricts AuNP loading capacity. Thus, a simple and effective strategy for realizing elevated AuNP accumulation at a test area is essential but remains a considerable challenge.

Recent studies have demonstrated that $\mathrm{Au}$ assemblies with numerous isolated AuNP can significantly increase optical absorption due to the collective molar extinctions of the whole AuNP (Liu et al., 2015; Ross et al., 2016; Shi et al., 2017; Yang et al., 2017). Currently available assemblies primarily include liposome (Sugikawa et al., 2016), mesoporous silica (Huang et al., 2019), polymersome (Qiao et al., 2018), and metal-organic framework (Huang et al., 2018c) because of their large surface areas and inner volumes. Our previous work reported the successful synthesis of a highly luminescent quantum dot (QD)-doped polymersome by encapsulating numerous individual QD into polymer matrix. The results indicated that the QD polymersome exhibited approximately 2,863-fold improvement in luminescent intensity compared with the corresponding QD. By using this QD polymersome as a signal reporter, the developed LFIA achieved an ultrasensitive detection of aflatoxin $\mathrm{B}_{1}$ with a limit of detection (LOD) of 0.42 $\mathrm{pg} / \mathrm{mL}$, approximately 2 orders of magnitude lower than the conventional LFIA based on 30-nm AuNP as labels (Ren et al., 2014).

Motivated by this previous study, novel AuNPdoped polymersomes, defined as gold superparticles (GSP), were synthesized herein by encapsulating numerous isolated AuNP into a poly(maleicanhydridealt-1-octadecene) (PMAO) polymer matrix using an evaporation-based self-assembly strategy. The synthesized GSP exhibited a regular quasi-spherical morphology with densely packed AuNP in the interior of the nanosphere. The GSP were further characterized with evidently enhanced optical absorption relative to the traditional 40-nm AuNP (AuNP 40 ). This characteristic contributes to increasing LFIA sensitivity. More importantly, the absorption of GSP was size dependent, where the absorption increased with increasing GSP size because more AuNP were embedded in large GSP than in small GSP. After covalent conjugation with antibodies, 342-nm GSP $\left(\mathbf{G S P}_{\mathbf{3 4 2}}\right)$ were successfully used as signal reporters for the enhanced detection of E. coli $\mathrm{O} 157: \mathrm{H} 7$ on a sandwich LFIA platform under optimized conditions. A LOD of $5.95 \times 10^{2} \mathrm{cfu} / \mathrm{mL}$ for E. coli $\mathrm{O} 157$ :H7 was approximately 32 -fold lower than that of the conventional LFIA strip with $\mathrm{AuNP}_{40}$ as labeling probes. In addition, the specificity, accuracy, reproducibility, reliability, and practicability of our proposed $\mathrm{GSP}_{342}$-LFIA were demonstrated in milk. The designed GSP can act as a promising signal reporter in LFIA to provide enhanced detection for various biomolecules, such as bacteria, viruses, and proteins.

\section{MATERIALS AND METHODS}

\section{Reagents}

The $\mathrm{HAuCl}_{4} \cdot 3 \mathrm{H}_{2} \mathrm{O}$, PMAO, SDS, oleamine, $N-(3-$ dimethylaminopropyl)- $N^{\prime}$-ethylcarbodiimide (EDC) hydrochloride, and BSA were purchased from Sigma-Aldrich Chemical (St. Louis, MO). A pair of mAb against E. coli O157:H7 was prepared as captured and detected antibody in our laboratory. Goat anti-mouse IgG was obtained from Chongqing Xinyuanjiahe Biotechnology Inc. (Chongqing, China). The NC membrane, absorbent pad, sample pad, and polyvinyl chloride backing card were obtained from Schleicher and Schuell GmbH (Dassel, Germany). The E. coli O157:H7-free whole milk samples were purchased from Carrefour Supermarket (Nanchang, China). Deionized water was purified using a Milli-Q system (Millipore, Milford, MA). All the other reagents were of analytical grade and purchased from Sinopharm Chemical Corp. (Shanghai, China).

\section{Instruments}

The UV-visible absorption spectra were recorded on a UV-visible spectrophotometer (G10S, Thermo Fisher, Waltham, MA). The size distribution of GSP was measured on a dynamic light scattering (DLS) analyzer (Malvern Nano ZSE, London, UK). The morphology of GSP was characterized on a high-resolution 
transmission electron microscope (TEM; JEOL JEM 2100, Tokyo, Japan).

\section{Bacterial Strains}

Listeria monocytogenes ATCC 13932, Salmonella choleraesuis ATCC 10708, Proteus vulgaris CMCC 49027, Bacillus subtilis collection, Listeria welshimeri ATCC 35897, Cronobacter muytjensii SR 1074B, Salmonella enteritidis ATCC 13076, Micrococcus luteus CMCC 28001, and Escherichia coli O157:H7 ATCC 43888 were kept in our laboratory and used in this work.

\section{Synthesis of OA-Capped AuNP}

Oleylamine (OA)-AuNP with a size of $10 \mathrm{~nm}$ were synthesized using a previously reported method (Hiramatsu and Osterloh, 2004). In particular, an $\mathrm{Au}^{3+}$ stock solution was prepared by mixing $0.3 \mathrm{mmol}$ of gold (III) chloride hydrate with $7.4 \mathrm{mmol}$ of $\mathrm{OA}$ in $1.0 \mathrm{~mL}$ of toluene. Then, the stock solution was added quickly into a boiling solution of $35.3 \mathrm{mmol}$ of OA dissolved in $49 \mathrm{~mL}$ of toluene under magnetic stirring. The color of the mixture solution immediately changed to bright yellow, and then to deep red. After $3 \mathrm{~h}, 50 \mathrm{~mL}$ of ethanol was added to precipitate the OA-AuNP. The obtained OA-AuNP were dried and stored for later use.

\section{Synthesis of GSP}

The GSP were synthesized as described previously with slight modifications (Duan et al., 2017). Briefly, $10 \mathrm{mg}$ of OA-AuNP was dissolved in $50 \mu \mathrm{L}$ of chloroform containing $2 \mathrm{mg}$ of PMAO. Then, the mixture was added to $250 \mu \mathrm{L}$ of SDS solution, followed by ultrasonic emulsification for $2 \mathrm{~min}$. After the chloroform was evaporated at $60^{\circ} \mathrm{C}$ for $3 \mathrm{~h}$, the GSP were centrifuged and resuspended in deionized water $(\mathrm{pH}=9)$ to hydrolyze the anhydride group of PMAO into the carboxyl group. Gold superparticles with other sizes were synthesized by adjusting the amount of SDS and ultrasonic power, as shown in Supplemental Table S1 (https://doi.org/10 .3168/jds.2019-17934).

\section{Preparation of GSP-mAb}

The GSP-mAb were prepared using an active ester method in the presence of EDC (Guo et al., 2019). In brief, anti- $E$. coli $\mathrm{O} 157: \mathrm{H} 7$ detected $\mathrm{mAb}(2 \mathrm{mg} / \mathrm{mL})$ and EDC at the desired amounts were added to $1 \mathrm{~mL}$ of GSP solution. After incubation for $90 \mathrm{~min}$ at room temperature, the reaction solution was blocked with $10 \%$ (wt/vol) skim milk. Finally, the obtained GSP-
$\mathrm{mAb}$ were collected via centrifugation and dispersed in $100 \mu \mathrm{L}$ of $\mathrm{PBS}(\mathrm{pH}=7.4)$ containing $1 \%$ BSA, $25 \%$ sucrose, and $0.1 \%$ Tween-20.

\section{Preparation of GSP-LFIA}

Similar to a conventional sandwich LFIA strip, GSPLFIA was composed of the following parts: sample pad, NC membrane, absorbent pad, and polyvinyl chloride backing card. Sample pads were treated with $20 \mathrm{mM}$ sodium borate buffer ( $\mathrm{pH}$ 8.0) containing 1.0\% (wt/ vol) BSA, $0.1 \%$ (wt/vol) $\mathrm{NaN}_{3}$, and $0.25 \%$ Tween-20. Anti-E. coli $\mathrm{O} 157: \mathrm{H} 7(1.5 \mathrm{mg} / \mathrm{mL})$ captured $\mathrm{mAb}$ and goat anti-mouse $\operatorname{IgG}(1.0 \mathrm{mg} / \mathrm{mL})$ were sprayed onto $\mathrm{NC}$ membranes as the test and control lines, respectively. The remaining procedures for strip assembly can be found in our previous work (Duan et al., 2019).

\section{Detection Procedures for E. coli 0157:H7 Using GSP $_{342^{2}-\text { LFIA }}$}

The $\mathrm{GSP}_{342}-\mathrm{mAb}(6 \mu \mathrm{L}, 0.44 \mathrm{fmol})$ was added into 64 $\mu \mathrm{L}$ of the sample solution with desired concentrations of E. coli $\mathrm{O} 157: \mathrm{H} 7$, and then the mixture was incubated for $3 \mathrm{~min}$. The reacted solution was pipetted into the sample well of the strip. After 15 min, the optical densities at the test $\left(\mathbf{O D}_{\mathbf{T}}\right)$ and control $\left(\mathbf{O D}_{\mathbf{C}}\right)$ lines were recorded using a commercial HG-8 strip reader (Shanghai Huguo Science Instrument Co. Ltd., Shanghai, China).

\section{Sensitivity}

A stock solution of E. coli O157:H7 with a concentration of $1.95 \times 10^{9} \mathrm{cfu} / \mathrm{mL}$ was serially diluted to $1.9 \times$ $10^{7}, 9.75 \times 10^{6}, 4.87 \times 10^{6}, 2.43 \times 10^{6}, 1.21 \times 10^{6}, 6.09$ $\times 10^{5}, 3.04 \times 10^{5}, 1.52 \times 10^{5}, 7.61 \times 10^{4}, 3.80 \times 10^{4}$, $1.90 \times 10^{4}, 9.52 \times 10^{3}, 4.76 \times 10^{3}, 2.38 \times 10^{3}, 1.19 \times$ $10^{3}, 5.95 \times 10^{2}, 2.97 \times 10^{2}$, and $0 \mathrm{cfu} / \mathrm{mL}$ in sterile 0.01 $M$ PBS solution containing 10-fold diluted milk. The prepared E. coli O157:H7-spiked milk solutions were then tested using our GSP $_{342}$-LFIA strip. The standard curve of this strip was constructed by plotting the $\mathrm{OD}_{\mathrm{T}} / \mathrm{OD}_{\mathrm{C}}$ value against the logarithmic concentrations of $E$. coli $\mathrm{O} 157: \mathrm{H} 7$.

\section{Specificity}

Eight nontarget strains at the concentrations of $10^{6}$ $\mathrm{cfu} / \mathrm{mL}$ were used to determine the specificity of GSPLFIA, including L. monocytogenes ATCC 13932, S. choleraesuis ATCC 10708, P. vulgaris CMCC 49027, L. welshimeri ATCC 35897, C. muytjensii SR 1074B, B. subtilis collection, $S$. enteritidis ATCC 13076, and $M$. luteus CMCC 28001. Meanwhile, E. coli O157:H7 (10 
$\mathrm{cfu} / \mathrm{mL}$ ) was used as a positive control, and a sterile blank PBS solution was applied as a negative control.

\section{Detection of E. coli 0157:H7 in Whole Milk Using GSP $_{342^{-}}$LFIA}

Whole milk $(25 \mathrm{~mL})$ without E. coli $\mathrm{O} 157: \mathrm{H} 7$ was mixed with $225 \mathrm{~mL}$ of sterilized Luria-Bertani medium and E. coli O157:H7 (6 cfu). The mixture was then incubated with gentle shaking at $150 \mathrm{rpm}$ at $37^{\circ} \mathrm{C}$. During the culture, $1 \mathrm{~mL}$ of the milk sample was transferred to a sterile plastic culture tube at different time points of $3.0,4.5,6.0,7.5,8.5,10.0$, and $11.0 \mathrm{~h}$, respectively. Last, the obtained samples were simultaneously measured using our prepared $\mathrm{GSP}_{342}$-LFIA strip and conventional AuNP-based strip.

\section{RESULTS AND DISCUSSION}

\section{Synthesis and Characterization of GSP}

The assembled GSP were synthesized using an evaporation-induced self-assembly method. The OA-AuNP (10 nm, Supplemental Figure S1; https://doi.org/10 $.3168 /$ jds.2019-17934) were used as the building blocks for GSP synthesis. The synthesis strategy for GSP is illustrated in Figure 1. The resultant GSP were further characterized by TEM, UV-visible absorption spectroscopy, and DLS analysis. The TEM images in Figure 2A and Supplemental Figures S2A-S2D (https://doi.org/ 10.3168/jds.2019-17934) show that the obtained GSP have a relatively uniform and regular spherical shape with sizes of $90,120,228,311$, and $500 \mathrm{~nm}$. The figures also show that numerous small OA-AuNP were embedded and evenly distributed in the interior of GSP. The number of embedded OA-AuNP evidently increased with GSP size. The DLS analysis showed that the average hydrodynamic diameters of GSP were slightly larger than those obtained in TEM images, primarily due to the polymer layer on the surface of GSP that exhibited low contrast in TEM imaging. Moreover, all GSP demonstrated low polydispersity indices of below 0.2 , indicating the high colloidal stability of GSP in a solution. The UV-visible spectrum analysis of GSP found that the maximum absorption peak produced a redshift from 531 to $564 \mathrm{~nm}$ with increasing the GSP size (Supplemental Figure S2E, https://doi.org/10 $.3168 / j d s .2019-17934)$, and the solution color changed from red to amaranth. The slight shift in the color of GSP does not compromise visual detection based on the naked eye. Notably, the absorbance of GSP presented an evident size-dependent increase. Compared

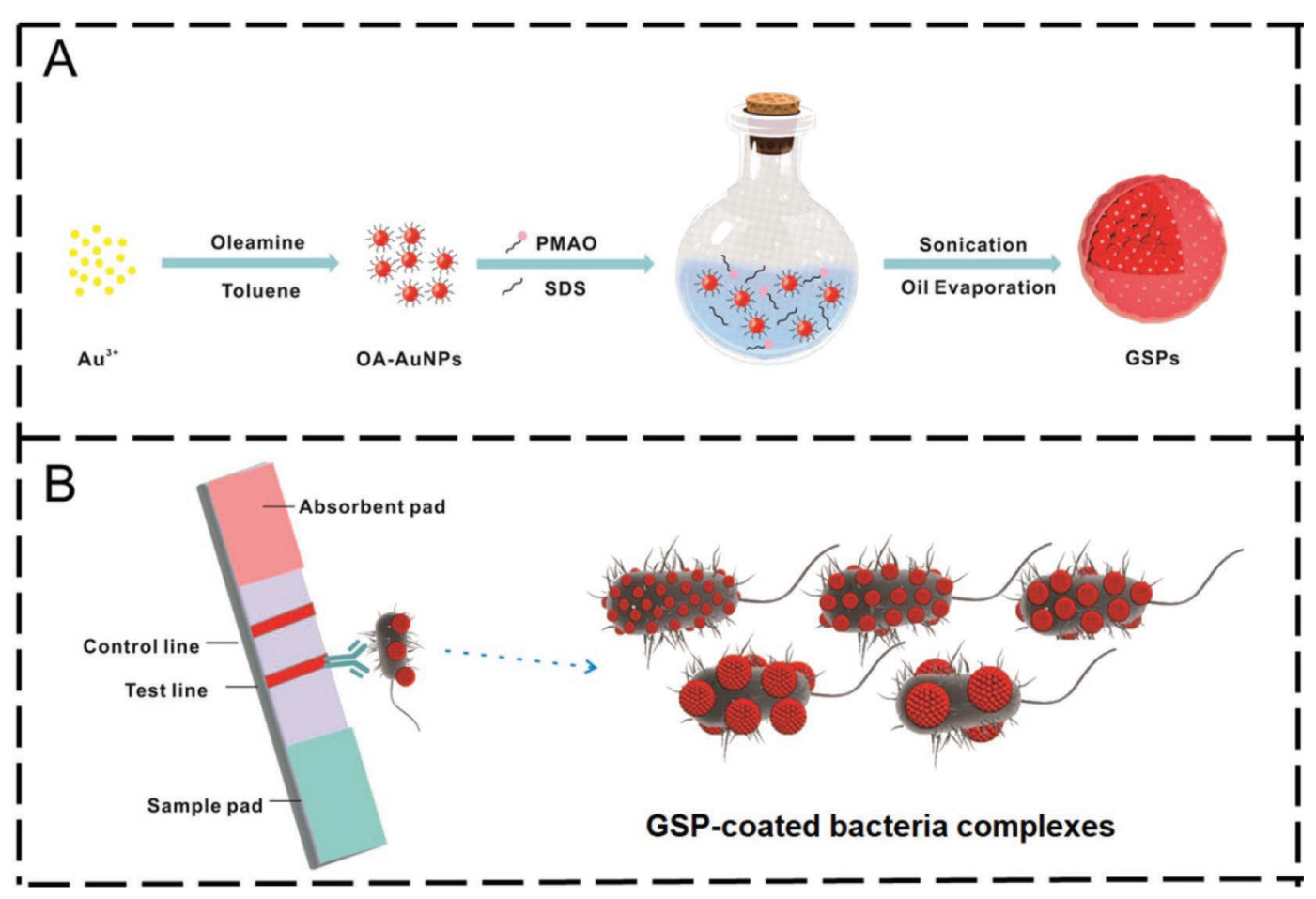

Figure 1. Schematic illustration of (A) the syntheses of gold superparticles (GSP). (B) Sandwich lateral flow immunoassay strips based on different-sized GSP as signal labels for detecting Escherichia coli O157:H7. PMAO = poly(maleicanhydride-alt-1-octadecene); OA = oleylamine. 
with the widely used $\mathrm{AuNP}_{40}$, the absorbance of the 5 synthesized GSP exhibited an apparent improvement ranging from 1.3- to 60.9-fold (Figure 2B), and thus theoretically contributed to enhancing the sensitivity of LFIA as signal reporters. In addition, we analyzed the relationship between GSP size and optical density value on the strip by spraying the $5 \mathrm{GSP}$ and $\mathrm{AuNP}_{40}$ on the NC membrane as test line at the same particle concentration. As shown in Figure $2 \mathrm{C}$, the $\mathrm{OD}_{\mathrm{T}}$ values gradually increased with increasing the GSP size, and the values were remarkably higher than those of $\mathrm{AuNP}_{40}$. These results further prove that using GSP to replace $\mathrm{AuNP}_{40}$ as labeling probes in LFIA is beneficial for achieving highly sensitive detection. The stability of GSP was evaluated by recording the changes of absorbance and size against $\mathrm{pH}$ value and buffer solutions. Results in Supplemental Figure S3A (https://doi .org/10.3168/jds.2019-17934) display that a negligible change is observed in the hydrodynamic diameter and the absorbance when the $\mathrm{pH}$ value ranges from 5 to 9 . In addition, Supplemental Figure S3B (https://doi.org/ 10.3168/jds.2019-17934) demonstrates that no obvious change is seen in the hydrodynamic diameter when the GSP were dispersed in buffer or milk solution. These results indicate excellent colloidal and optical stability of our GSP and good dispersion in buffer or milk, which contributes to the successful sensing application. Encouraged by this, we further investigated the feasibility of using the GSP as enhanced signal probes for improving E. coli O157:H7 detection on the LFIA platform.

\section{Synthesis and Optimization of GSP-mAb}

Before LFIA detection, GSP were functionalized with antibodies against E. coli O157:H7. Several key factors influencing the conjugation efficiency and an- tibody activity, including $\mathrm{pH}$ value, EDC amount, and labeled amount of anti- $E$. coli $\mathrm{O} 157: \mathrm{H} 7$ detected $\mathrm{mAb}$ were optimized, as shown in Supplemental Figures S4 to S8 (https://doi.org/10.3168/jds.2019-17934). The condition that can provide the highest $\mathrm{OD}_{\mathrm{T}}$ value at an E. coli $\mathrm{O} 157: \mathrm{H} 7$ concentration of $10^{6} \mathrm{cfu} / \mathrm{mL}$ was considered optimal. On the basis of this standard, the selected condition combinations for the 5 GSP sizes are summarized in Supplemental Table S2 (https://doi.org/ 10.3168/jds.2019-17934). As indicated in Supplemental Table S2, the optimal $\mathrm{pH}$ values for $\mathrm{GSP}_{98}, \mathrm{GSP}_{144}$, $\mathrm{GSP}_{253}, \mathrm{GSP}_{342}$, and $\mathrm{GSP}_{547}$ were 5.5, 6.0, 5.5, 5.5, and 7.5 , respectively. The optimal EDC concentrations for $\mathrm{GSP}_{98}, \mathrm{GSP}_{144}, \mathrm{GSP}_{253}, \mathrm{GSP}_{342}$, and $\mathrm{GSP}_{547}$ solutions were $5.0,5.0,5.0,2.0$, and $2.0 \mu \mathrm{g} / \mathrm{mL}$, respectively. The optimal labeled amounts of anti-E. coli O157:H7 detected $\mathrm{mAb}$ were 15.0, 21.0, 110.0, 184.0, and $565.4 \mu \mathrm{g}$ per pmol GSP $98, \mathrm{GSP}_{144}, \mathrm{GSP}_{253}, \mathrm{GSP}_{342}$, and $\mathrm{GSP}_{547}$, respectively.

The concentration of anti- $E$. coli O157:H7 captured $\mathrm{mAb}$ sprayed on the test line and the amount of GSP$\mathrm{mAb}$ used in each strip were another 2 key variables that can affect LFIA sensitivity. Thus, we further optimized these 2 factors by detecting the same concentration of E. coli $\mathrm{O} 157: \mathrm{H} 7\left(10^{6} \mathrm{cfu} / \mathrm{mL}\right)$ using the corresponding GSP-LFIA strips. The results in Supplemental Figures S4D to S8D and S9 (https://doi.org/10.3168/jds.2019 -17934) revealed that the optimal concentration of anti-E. coli $\mathrm{O} 157: \mathrm{H} 7 \mathrm{mAb}$ sprayed on the test line was $1.5 \mathrm{mg} / \mathrm{mL}$, and the optimal amounts of $\mathrm{GSP}_{98}-\mathrm{mAb}$, $\mathrm{GSP}_{144}-\mathrm{mAb}, \mathrm{GSP}_{253}-\mathrm{mAb}, \mathrm{GSP}_{342^{2}}-\mathrm{mAb}$, and $\mathrm{GSP}_{547^{-}}$ $\mathrm{mAb}$ probes used in each strip were 9.4, 2.8, 1.1, 1.4, and $0.2 \mathrm{fmol}$, respectively. It should be pointed out that an evident background color for GSP $_{547}$ was observed during the entire optimization process. A possible reason for such a result is discussed in detail below.
A

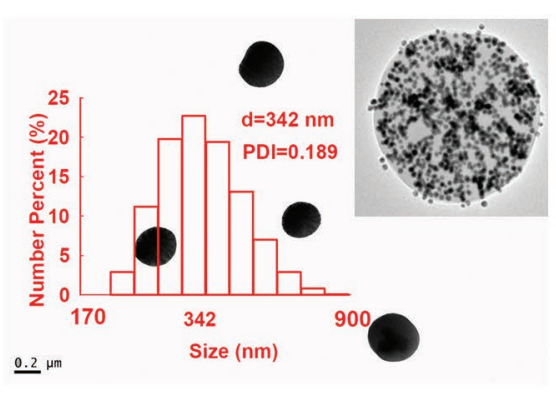

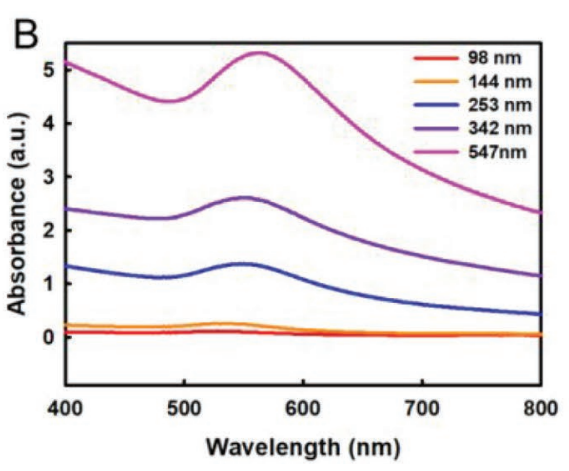

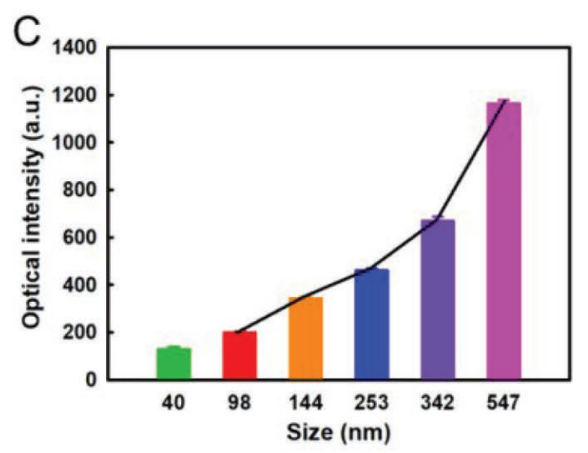

Figure 2. Characterization of gold superparticles (GSP). (A) Transmission electron microscope image and dynamic light scattering analysis for 342-nm GSP $\left(\mathrm{GSP}_{342}\right)$, where d = diameter and PDI = polydispersity index. (B) Ultraviolet-visible absorption spectra of 5 different-sized GSP at the same particle concentration. (C) Optical intensities at the test area of test strip sprayed using 5 different-sized GSP at the same particle concentration. a.u. = arbitrary units. 


\section{Effect of the GSP Size on the LFIA Sensitivity in Detecting E. coli 0157:H7}

For the sandwich GSP-LFIA detection of bacteria, the analytical sensitivity is typically determined using the following factors: (1) the inherent optical signal intensity of individual GSP, (2) the binding number of the GSP probes on bacterial surface, and (3) the diffusion rates of the GSP probes on the NC membrane. Our previous result proved that the optical signal intensity of individual GSP is closely related to their size. That is, when the GSP size is large, its absorbance is high. Thus, the use of larger-sized GSP as LFIA labels can theoretically increase sensitivity. However, largersized GSP typically decrease GSP binding number at a given bacterial surface. Escherichia coli O157:H7 exhibits larger sizes $(0.5 \mu \mathrm{m} \times 1$ to $3 \mu \mathrm{m})$ and contains more antigen epitopes on their surfaces. Such unique properties make it highly suitable for binding with GSP probes to form a GSP-coated bacteria complex, as indicated in Figure 3A. The theoretical maximum binding number of the GSP on the surface of $E$. coli $\mathrm{O} 157: \mathrm{H} 7$ was simulated in accordance with the corresponding geometric sizes of both. The simulation results are pre-
A

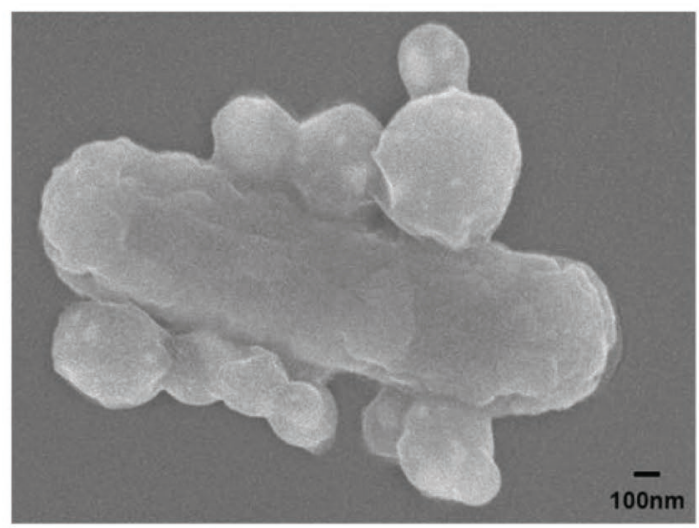

C

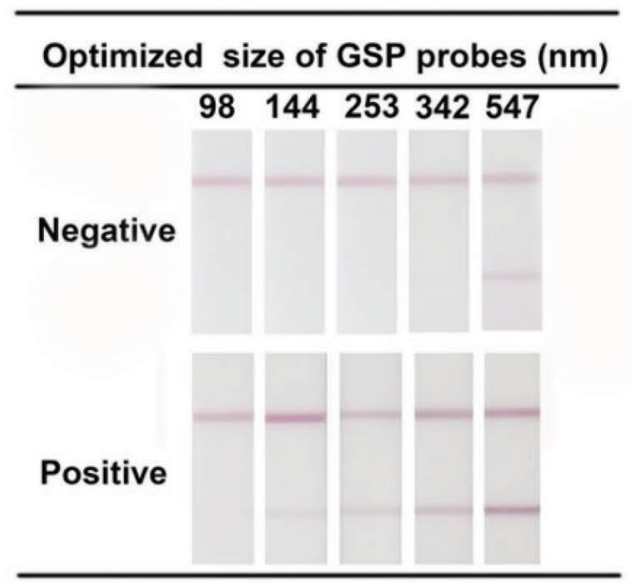

B
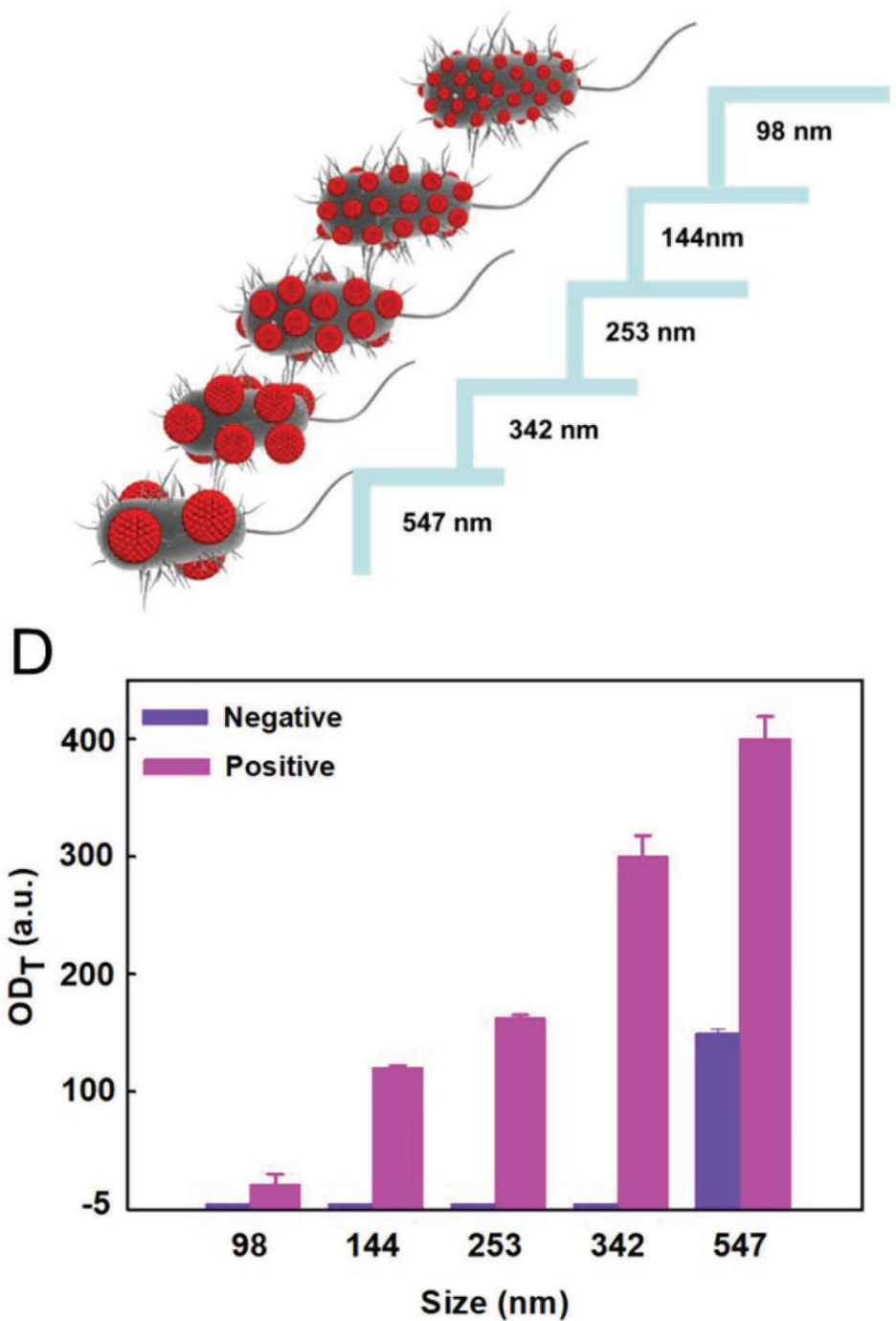

Figure 3. Characterization of gold superparticle (GSP)-coated bacteria complexes with 5 different sizes of GSP probes and optimization of the size of the GSP probes in lateral flow immunoassay. (A) Scanning electron microscope image of GSP ${ }_{342}$-coated bacteria complexes. (B) Simulation of 5 different-sized GSP bound with Escherichia coli O157:H7. (C) The prototypes of test strips based on 5 different-sized GSP after reaction with $E$. coli $\mathrm{O} 157: \mathrm{H} 7$ at the same concentration of $3.8 \times 10^{4} \mathrm{cfu} / \mathrm{mL}$. (D) The corresponding optical density values at the test line $\left(\mathrm{OD}_{\mathrm{T}}\right)$ of 5 reacted strips in Figure 2C. a.u. = arbitrary units. Error bars indicate the SD from 3 independent measurements. 
sented in Figure 3B, in which the binding number of GSP on the bacterial surface was markedly reduced with increasing GSP size to result in a decreased signal output. Such reduction occurred because the oversized GSP resulted in fewer GSP probes bound on the bacterial surface. Meanwhile, a large size can cause the slower diffusion of GSP on the $\mathrm{NC}$ membrane due to the reduced Brownian motion, thereby giving rise to the low immunoreaction efficiency between GSP-mAbbacteria complex and the captured $\mathrm{mAb}$ pre-sprayed on the test line for decreased GSP capture to produce low colorimetric signal intensity. On the basis of the preceding analysis, GSP with appropriate size is necessary to achieve the highest sensitivity of LFIA in detecting of E. coli O157:H7. Therefore, we further investigated the effect of GSP size on the sensitivity of LFIA under their respective optimal conditions. To achieve this objective, the E. coli O157:H7-spiked milk samples at a concentration of $3.8 \times 10^{4} \mathrm{cfu} / \mathrm{mL}$ (this concentration was the visual LOD value obtained using $\mathrm{GSP}_{98}$ as labels) were measured using the 5 different-sized GSP-based LFIA strips. The results in Figure 3C and $3 \mathrm{D}$ revealed that the $\mathrm{OD}_{\mathrm{T}}$ value increased significantly with increasing the size of GSP probes. However, the $\mathrm{GSP}_{547}$ probes exhibited a distinct nonspecific binding for high background signals that was not observed in the GSP probes with other sizes. The possible reason for this finding is that the larger size of GSP (e.g., $547 \mathrm{~nm}$ ) resulted in low diffusion rates and high probability to settle in the membrane pores, thus increasing background residue on the test line. By using $\Delta O D_{T}$, which represents the $\mathrm{OD}_{\mathrm{T}}$ value difference between the positive and negative samples, $\mathrm{GSP}_{342}$ provided the highest $\Delta \mathrm{OD}_{\mathrm{T}}$ among these 5 GSP sizes. Therefore,
A
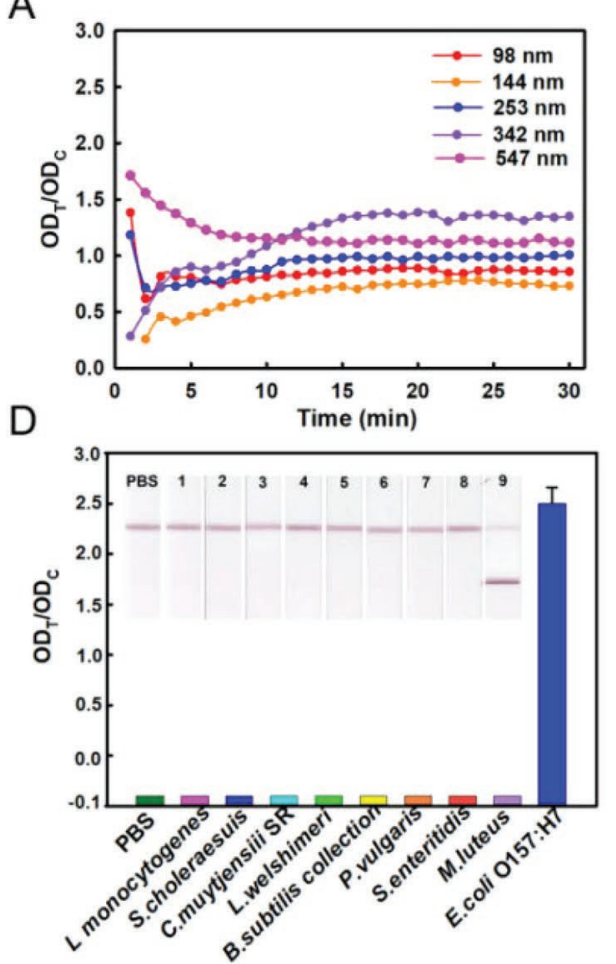

B

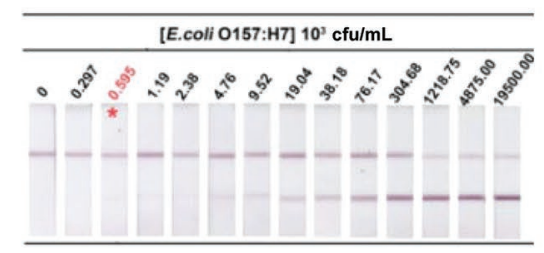

$E$

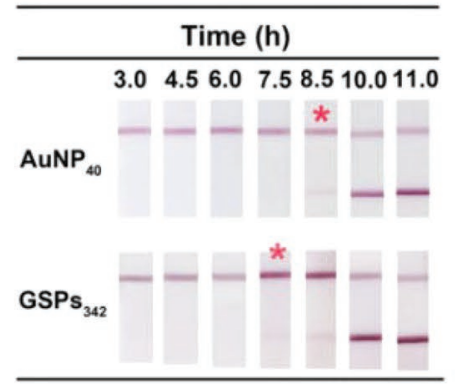

C

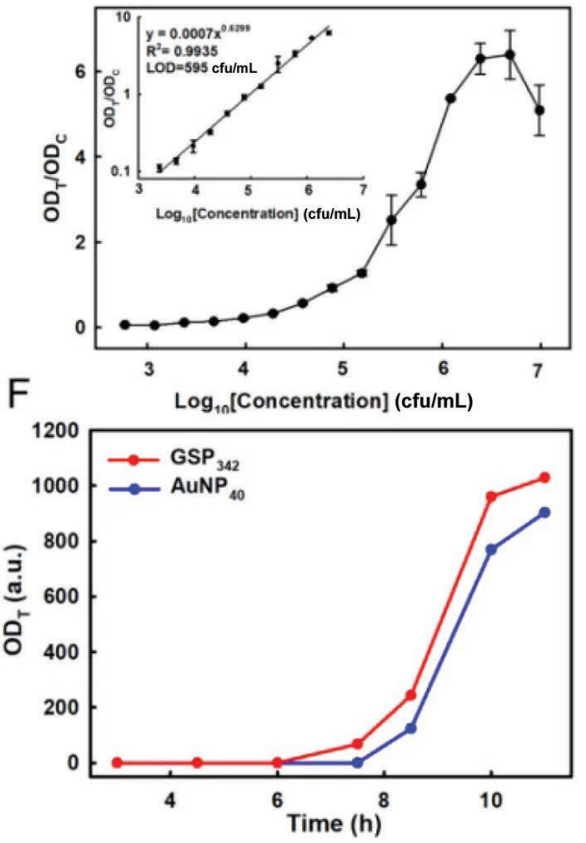

Figure 4. Sensitivity and selectivity analysis of our proposed 342-nm gold superparticle $\left(\mathrm{GSP}_{342}\right)$ based lateral flow immunoassay (LFIA) in Escherichia coli O157:H7 spiked milk samples. (A) Immunoreaction dynamic analysis of gold superparticle (GSP) with 5 different sizes. (B) The strip prototypes after detecting E. coli O157:H7 at different concentrations ranging from 0 to $1.9 \times 10^{7} \mathrm{cfu} / \mathrm{mL}$ in whole milk. (C) Construction of the standard curves by plotting the logarithmic $\mathrm{OD}_{\mathrm{T}} / \mathrm{OD}_{\mathrm{C}}$ value against the logarithmic E. coli O157:H7 concentration in whole milk, where $\mathrm{OD}_{\mathrm{T}}$ is the optical density at the test line and $\mathrm{OD}_{\mathrm{C}}$ is the optical density at the control line. (D) Specificity experiment between $E$. coli O157:H7 and nontarget bacteria. Numbers 1 to 9: Listeria monocytogenes ATCC 13932, Salmonella choleraesuis ATCC 10708, Proteus vulgaris CMCC 49027, Listeria welshimeri ATCC 35897, Cronobacter muytjensii SR 1074B, Bacillus subtilis collection, Salmonella enteritidis ATCC 13076, Micrococcus luteus CMCC 28001, and E. coli O157:H7 ATCC 43888. (E) The strip prototypes after detecting E. coli O157:H7 at different incubation times using the GSP ${ }_{342}$-LFIA strip. (F) The corresponding optical density values at the test lines from Figure 3E. Meanwhile, a conventional $40 \mathrm{~nm}$ AuNP-LFIA strip was used as a comparison with our proposed GSP $_{342}$-LFIA. LOD = limit of detection. Error bars indicate the SD from 3 independent measurements. The asterisk in panel B indicates the LOD of the GSP342-LFIA strip for E. coli O157:H7 detection. The asterisks in panel E indicate the shortest incubation time for the positive detection of E. coli O157:H7 using GSP342-LFIA strip and AuNP40-LFIA, respectively. 
GSP $_{342}$ probes were selected as the LFIA signal reporters for detecting E. coli O157:H7 in all the succeeding experiments.

\section{Performance Evaluation of GSP $_{342}$-LFIA in Milk}

Immunological kinetics analysis was then applied to optimize the signal readout time of $\mathrm{GSP}_{342}$-LFIA. Results in Figure $4 \mathrm{~A}$ indicated that the $\mathrm{OD}_{\mathrm{T}} / \mathrm{OD}_{\mathrm{C}}$ value reached a plateau after $15 \mathrm{~min}$, implying that $15 \mathrm{~min}$ of immunoreaction after sample addition can enable a reliable, robust, and highly reproducible LFIA detection. Under optimal conditions, the GSP $_{342}$-LFIA strip was then extended to detect a series of milk samples with desired concentrations of $3.0 \times 10^{2}$ to $1.9 \times 10^{7}$ $\mathrm{cfu} / \mathrm{mL}$. Figure $4 \mathrm{~B}$ shows that the red band at the test line gradually deepened with the increase of $E$. coli O157:H7 concentration and the visual LOD value of this method reached up to $4.76 \times 10^{3} \mathrm{cfu} / \mathrm{mL}$. Further standard curve was constructed by plotting the logarithmic $\mathrm{OD}_{\mathrm{T}} / \mathrm{OD}_{\mathrm{C}}$ value against the logarithmic $E$. coli O157:H7 concentration (Figure 4C). The regression equation can be expressed as $y=0.0007 \times x^{0.6299}$ $\left(\mathrm{R}^{2}=0.9935\right)$. The LOD value of our $\mathrm{GSP}_{342}$-LFIA was as low as $5.95 \times 10^{2} \mathrm{cfu} / \mathrm{mL}$, which was the lowest concentration of $E$. coli $\mathrm{O} 157: \mathrm{H} 7$ that could cause an apparent $\mathrm{OD}_{\mathrm{T}}$ value recorded by the strip reader. The LOD value was approximately 32 times lower than that of traditional $\mathrm{AuNP}_{40}$-LFIA strip $(\mathrm{LOD}=1.90 \times$ $10^{4} \mathrm{cfu} / \mathrm{mL}$, Supplemental Figures S10 and S11; https: //doi.org/10.3168/jds.2019-17934). In addition, the $\mathrm{GSP}_{342}$-LFIA strip also displayed a wider linear detection range of $2.38 \times 10^{3}$ to $2.43 \times 10^{6} \mathrm{cfu} / \mathrm{mL}$ compared with the traditional $\mathrm{AuNP}_{40}$-LFIA strip (the linear detection ranged from $3.80 \times 10^{4}$ to $\left.2.43 \times 10^{6} \mathrm{cfu} / \mathrm{mL}\right)$. More importantly, the LOD value of our strip method was comparable with and even superior to the previously reported strip methods (Supplemental Table S3; https://doi.org/10.3168/jds.2019-17934). These results demonstrated the feasibility of using our designed GSP to improve the sensitivity of LFIA with a sandwich format.

Selectivity was further estimated by determining 8 other common foodborne pathogens in milk samples using the developed $\mathrm{GSP}_{342}$-LFIA method. Meanwhile, E. coli $\mathrm{O} 157: \mathrm{H} 7$ was used as the positive control and sterile PBS was used as the negative control. Figure $4 \mathrm{D}$ shows that a clear red band at the test line with an evident increase in $\mathrm{OD}_{\mathrm{T}} / \mathrm{OD}_{\mathrm{C}}$ value was only observed when detecting E. coli O157:H7. By contrast, negligible responses in color and $\mathrm{OD}_{\mathrm{T}} / \mathrm{OD}_{\mathrm{C}}$ value were obtained when testing other nontarget bacteria. This finding suggested the excellent specificity of our method against E. coli $\mathrm{O} 157: \mathrm{H} 7$. The accuracy and precision of our $\mathrm{GSP}_{342}$-LFIA strip was evaluated through an addition and recovery experiment at the $E$. coli $\mathrm{O} 157: \mathrm{H} 7$ concentrations of $4.7 \times 10^{3}, 1.9 \times 10^{4}, 7.6 \times 10^{4}$, and $1.5 \times 10^{5} \mathrm{cfu} / \mathrm{mL}$ (Table 1 ). The average recoveries for intra- and interassays ranged from 83 to $112 \%$ with the coefficient of variation ranging from 5.1 to $10 \%$. The accuracy and precision of the developed $\mathrm{GSP}_{342^{-}}$ LFIA are acceptable for quantitative determination of E. coli O157:H7 in milk samples. Furthermore, the reliability and practicability were detected by testing 28 E. coli O157:H7-spiked milk samples with known concentrations ranging of $9.76 \times 10^{2}$ to $1.00 \times 10^{6} \mathrm{cfu} /$ $\mathrm{mL}$ confirmed using the plate counting method. The results in Table 2 revealed that the average recoveries for all samples varied from 81 to $120 \%$ with the coefficient of variation below 10\%, proving the applicability of our method for E. coli $\mathrm{O} 157: \mathrm{H} 7$ detection in real milk samples.

\section{Detection of E. coli 0157:H7-Spiked Milk}

Escherichia coli O157:H7 with a bacterial concentration of $6 \mathrm{cfu}$ was inoculated into a real milk sample. The inoculated milk sample was incubated and detected using our GSP $_{342}$-LFIA strip at 3.0, 4.5, 6.0, 7.5, 8.5, 10.0, and $11.0 \mathrm{~h}$. Figure $4 \mathrm{E}$ and $4 \mathrm{~F}$ exhibited that

Table 1. Recovery experiment for detection of Escherichia coli O157:H7 in whole milk based on gold superparticle lateral flow immunoassay

\begin{tabular}{|c|c|c|c|}
\hline $\begin{array}{l}\text { Spiked E. coli } \\
\text { O157:H7 (cfu/mL) }\end{array}$ & $\begin{array}{c}\text { Mean } \\
(\mathrm{cfu} / \mathrm{mL})\end{array}$ & $\begin{array}{l}\text { Recovery } \\
(\%)\end{array}$ & $\begin{array}{l}\mathrm{CV} \\
(\%)\end{array}$ \\
\hline $9.76 \times 10^{2}$ & $11.79 \times 10^{2}$ & 120 & 9.5 \\
\hline $1.95 \times 10^{3}$ & $2.07 \times 10^{3}$ & 106 & 7.0 \\
\hline $3.12 \times 10^{3}$ & $3.00 \times 10^{3}$ & 96 & 7.4 \\
\hline $3.90 \times 10^{3}$ & $3.75 \times 10^{3}$ & 96 & 10.0 \\
\hline $4.76 \times 10^{3}$ & $4.72 \times 10^{3}$ & 99 & 7.4 \\
\hline $6.25 \times 10^{3}$ & $5.08 \times 10^{3}$ & 81 & 4.3 \\
\hline $7.80 \times 10^{3}$ & $6.69 \times 10^{3}$ & 85 & 8.1 \\
\hline $9.50 \times 10^{3}$ & $9.82 \times 10^{3}$ & 103 & 5.0 \\
\hline $1.25 \times 10^{4}$ & $1.14 \times 10^{4}$ & 91 & 5.5 \\
\hline $1.56 \times 10^{4}$ & $1.52 \times 10^{4}$ & 97 & 10.0 \\
\hline $1.90 \times 10^{4}$ & $1.92 \times 10^{4}$ & 101 & 6.4 \\
\hline $2.50 \times 10^{4}$ & $2.13 \times 10^{4}$ & 85 & 2.0 \\
\hline $3.12 \times 10^{4}$ & $2.75 \times 10^{4}$ & 88 & 10 \\
\hline $3.80 \times 10^{4}$ & $3.40 \times 10^{4}$ & 89 & 2.5 \\
\hline $5.00 \times 10^{4}$ & $4.96 \times 10^{4}$ & 99 & 5.6 \\
\hline $6.25 \times 10^{4}$ & $5.35 \times 10^{4}$ & 85 & 3.5 \\
\hline $7.61 \times 10^{4}$ & $8.16 \times 10^{4}$ & 107 & 7.0 \\
\hline $1.00 \times 10^{5}$ & $1.11 \times 10^{5}$ & 111 & 6.0 \\
\hline $1.25 \times 10^{5}$ & $1.11 \times 10^{5}$ & 89 & 4.0 \\
\hline $1.52 \times 10^{5}$ & $1.36 \times 10^{5}$ & 89 & 3.5 \\
\hline $2.00 \times 10^{5}$ & $1.89 \times 10^{5}$ & 94 & 3.1 \\
\hline $2.50 \times 10^{5}$ & $2.34 \times 10^{5}$ & 93 & 6.4 \\
\hline $3.04 \times 10^{5}$ & $2.46 \times 10^{5}$ & 81 & 3.5 \\
\hline $4.00 \times 10^{5}$ & $4.19 \times 10^{5}$ & 105 & 9.5 \\
\hline $5.00 \times 10^{5}$ & $5.26 \times 10^{5}$ & 105 & 7.2 \\
\hline $6.09 \times 10^{5}$ & $5.27 \times 10^{5}$ & 86 & 3.0 \\
\hline $8.00 \times 10^{5}$ & $7.93 \times 10^{5}$ & 99 & 5.6 \\
\hline $1.00 \times 10^{6}$ & $9.85 \times 10^{5}$ & 98 & 6.9 \\
\hline
\end{tabular}


Table 2. Recoveries and precision for detection of Escherichia coli O157:H7 by gold superparticle lateral flow immunoassay in whole milk

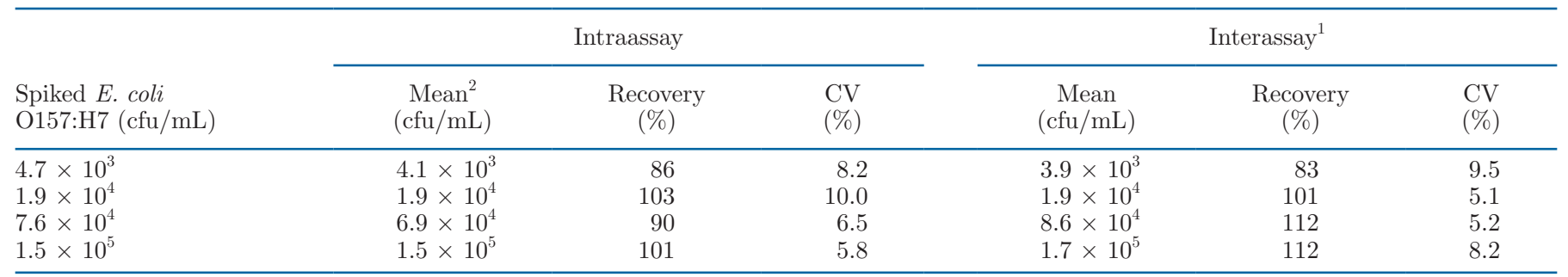

${ }^{1}$ Assay was completed every $1 \mathrm{~d}$ for $3 \mathrm{~d}$ continuously.

${ }^{2}$ Mean value of 5 replicates at each spiked concentration.

the time for the positive detection of E. coli O157:H7 in milk was $8.5 \mathrm{~h}$ using a conventional $\mathrm{AuNP}_{40}$-LFIA strip, whereas a shorter time of $7.5 \mathrm{~h}$ was required to produce the positive detection using our enhanced GSP $_{342}$-LFIA strip.

\section{CONCLUSIONS}

We successfully synthesized a novel GSP nanostructure using a simple evaporation-induced self-assembly strategy. The obtained GSP exhibit a typical spherical shape with numerous compactly packed AuNP in their interior. Further characterization of optical properties demonstrated significantly increased absorbance compared with the widely used $\mathrm{AuNP}_{40}$, and the absorbance of GSP can be easily tuned by changing their size. After covalent conjugation with antibodies, the GSP indicated an evident size-dependent change in the LFIA sensitivity and achieved the best detection for E. coli $\mathrm{O} 157: \mathrm{H} 7$ with a LOD of $5.95 \times 10^{2} \mathrm{cfu} / \mathrm{mL}$ in milk when using GSP $_{342}$ as LFIA signal reporters. The LOD value is approximately 32 -fold lower than that of the traditional AuNP $40^{-}$-based LFIA. Additionally, the specificity, accuracy, reliability, and practicability of our method were demonstrated in a real milk sample. Overall, this work presents a new method for rapid and sensitive screening of E. coli O157:H7 in milk, and offers a new strategy for improving the LFIA sensitivity using GSP as enhanced labels.

\section{ACKNOWLEDGMENTS}

This work was supported by a grant from the National Key Research and Development Program of China (Beijing; 2018YFC1602202 and 2018YFC1602505), the National Basic Research Program of China (Beijing; 2013CB127804), and the Major Projects of Natural Science Foundation of Jiangxi Province (Nanchang, China; 20161ACB20002). The authors have not stated any conflicts of interest.

\section{REFERENCES}

Chen, X., Y. Leng, L. Hao, H. Duan, J. Yuan, W. Zhang, X. Huang, and Y. Xiong. 2020. Self-assembled colloidal gold superparticles to enhance the sensitivity of lateral flow immunoassays with sandwich format. Theranostics 10:3737-3748. https://doi.org/10.7150/ thno.42364.

Choi, D. H., S. K. Lee, Y. K. Oh, B. W. Bae, S. D. Lee, S. Kim, Y.-B. Shin, and M.-G. Kim. 2010. A dual gold nanoparticle conjugatebased lateral flow assay (LFA) method for the analysis of troponin I. Biosens. Bioelectron. 25:1999-2002. https://doi.org/10.1016/j .bios.2010.01.019.

Deisingh, A. K., and M. Thompson. 2004. Strategies for the detection of Escherichia coli O157: H7 in foods. J. Appl. Microbiol. 96:419429. https://doi.org/10.1111/j.1365-2672.2003.02170.x.

Duan, H., X. Huang, Y. Shao, L. Zheng, L. Guo, and Y. Xiong. 2017. Size-dependent immunochromatographic assay with quantum dot nanobeads for sensitive and quantitative detection of ochratoxin A in corn. Anal. Chem. 89:7062-7068. https://doi.org/10.1021/acs .analchem.7b00869.

Duan, H., Y. Li, Y. Shao, X. Huang, and Y. Xiong. 2019. Multicolor quantum dot nanobeads for simultaneous multiplex immunochromatographic detection of mycotoxins in maize. Sens. Actuators B Chem. 291:411-417. https://doi.org/10.1016/j.snb.2019.04.101.

Guo, L., Y. Shao, H. Duan, W. Ma, Y. Leng, X. Huang, and Y. Xiong. 2019. Magnetic quantum dot nanobead-based fluorescent immunochromatographic assay for the highly sensitive detection of aflatoxin $B_{1}$ in dark soy sauce. Anal. Chem. 91:4727-4734. https: /doi.org/10.1021/acs.analchem.9b00223.

Guo, Q., J.-J. Han, S. Shan, D.-F. Liu, S.-S. Wu, Y.-H. Xiong, and W.-H. Lai. 2016. DNA-based hybridization chain reaction and biotin-streptavidin signal amplification for sensitive detection of Escherichia coli O157: H7 through ELISA. Biosens. Bioelectron. 86:990-995. https://doi.org/10.1016/j.bios.2016.07.049.

Hiramatsu, H., and F. E. Osterloh. 2004. A simple large-scale synthesis of nearly monodisperse gold and silver nanoparticles with adjustable sizes and with exchangeable surfactants. Chem. Mater. 16:2509-2511. https://doi.org/10.1021/cm049532v.

Hu, J., L. Wang, F. Li, Y. L. Han, M. Lin, T. J. Lu, and F. Xu. 2013. Oligonucleotide-linked gold nanoparticle aggregates for enhanced sensitivity in lateral flow assays. Lab Chip 13:4352-4357. https:// doi.org/10.1039/c3lc50672j.

Huang, H., G. Zhao, and W. Dou. 2018a. Portable and quantitative point-of-care monitoring of Escherichia coli O157: H7 using a personal glucose meter based on immunochromatographic assay. Biosens. Bioelectron. 107:266-271. https://doi.org/10.1016/j.bios .2018.02.027.

Huang, L., J. Jin, J. Wang, C. Jiang, M. Xu, H. Wen, T. Liao, and J. Hu. 2019. Homogeneous and high-density gold unit implanted optical labels for robust and sensitive point-of-care drug detection. Nanoscale 11:16026-16035. https://doi.org/10.1039/C9NR03740C.

Huang, Q., T. Bu, W. Zhang, L. Yan, M. Zhang, Q. Yang, L. Huang, B. Yang, N. Hu, Y. Suo, J. Wang, and D. Zhang. 2018b. An improved clenbuterol detection by immunochromatographic assay 
with bacteria@Au composite as signal amplifier. Food Chem. 262:48-55. https://doi.org/10.1016/j.foodchem.2018.04.085.

Huang, X., Z. He, D. Guo, Y. Liu, J. Song, B. C. Yung, L. Lin, G. Yu, J.-J. Zhu, Y. Xiong, and X. Chen. 2018c. "Three-in-one" nanohybrids as synergistic nanoquenchers to enhance no-wash fluorescence biosensors for ratiometric detection of cancer biomarkers. Theranostics 8:3461-3473. https://doi.org/10.7150/thno.25179.

Huang, Z., X. Cui, Q. Xie, D. Liu, and W. Lai. 2016. A novel method using immunomagnetic separation with a fluorescent nanobeads lateral flow assay for the rapid detection of low-concentration Escherichia coli O157: H7 in raw milk. J. Dairy Sci. 99:9581-9585. https://doi.org/10.3168/jds.2016-11780.

Jiang, H., W. Zhang, J. Li, L. Nie, K. Wu, H. Duan, and Y. Xiong. 2018. Inner-filter effect based fluorescence-quenching immunochromotographic assay for sensitive detection of aflatoxin $\mathrm{B}_{1}$ in soybean sauce. Food Control 94:71-76. https://doi.org/10.1016/j foodcont.2018.06.030.

Jiang, T., Y. Song, T. Wei, H. Li, D. Du, M.-J. Zhu, and Y. Lin. 2016. Sensitive detection of Escherichia coli O157: H7 using Pt-Au bimetal nanoparticles with peroxidase-like amplification. Biosens. Bioelectron. 77:687-694. https://doi.org/10.1016/j.bios.2015.10 .017 .

Lin, J., M. P. Smith, K. C. Chapin, H. S. Baik, G. N. Bennett, and J. W. Foster. 1996. Mechanisms of acid resistance in enterohemorrhagic Escherichia coli. Appl. Environ. Microbiol. 62:3094-3100. https://doi.org/10.1128/AEM.62.9.3094-3100.1996.

Liu, D., C. Li, F. Zhou, T. Zhang, H. Zhang, X. Li, G. Duan, W. Cai, and Y. Li. 2015. Rapid synthesis of monodisperse Au nanospheres through a laser irradiation-induced shape conversion, self-assembly and their electromagnetic coupling SERS enhancement. Sci. Rep. 5:7686. https://doi.org/10.1038/srep07686.

Liu, J., D. Mazumdar, and Y. Lu. 2006. A simple and sensitive "dipstick" test in serum based on lateral flow separation of aptamerlinked nanostructures. Angew. Chem. Int. Ed. Engl. 45:7955-7959. https://doi.org/10.1002/anie.200603106.

Luo, K., L. Hu, Q. Guo, C. Wu, S. Wu, D. Liu, Y. Xiong, and W. Lai. 2017. Comparison of 4 label-based immunochromatographic assays for the detection of Escherichia coli O157: H7 in milk. J. Dairy Sci. 100:5176-5187. https://doi.org/10.3168/jds.2017-12554.

Mead, P. S., and P. M. Griffin. 1998. Escherichia coli O157: H7. Lancet 352:1207-1212. https://doi.org/10.1016/S0140-6736(98)01267-7.

Qiao, X., B. Su, C. Liu, Q. Song, D. Luo, G. Mo, and T. Wang. 2018. Selective surface enhanced raman scattering for quantitative detection of lung cancer biomarkers in superparticle@MOF structure. Adv. Mater. 30:1702275. https://doi.org/10.1002/adma 201702275 .

Qiu, W., H. Xu, S. Takalkar, A. S. Gurung, B. Liu, Y. Zheng, Z. Guo, M. Baloda, K. Baryeh, and G. Liu. 2015. Carbon nanotube-based lateral flow biosensor for sensitive and rapid detection of DNA sequence. Biosens. Bioelectron. 64:367-372. https://doi.org/10 $.1016 /$ j.bios.2014.09.028.

Ren, M., H. Xu, X. Huang, M. Kuang, Y. Xiong, H. Xu, Y. Xu, H. Chen, and A. Wang. 2014. Immunochromatographic assay for ultrasensitive detection of aflatoxin $\mathrm{B}_{1}$ in maize by highly luminescent quantum dot beads. ACS Appl. Mater. Interfaces 6:1421514222. https://doi.org/10.1021/am503517s.

Ross, M. B., C. A. Mirkin, and G. C. Schatz. 2016. Optical properties of one-, two-, and three-dimensional arrays of plasmonic nanostructures. J. Phys. Chem. C 120:816-830. https://doi.org/ 10.1021/acs.jpcc.5b10800.

Samad, A., F. Abbas, Z. Ahmad, Z. Tanveer, I. Ahmad, S. G. Patching, N. Nawaz, M. T. Asmat, A. Raziq, M. Asadullah, M. A. Naeem, O. Akhtar, Pokryshko, and M. Z. Mustafa. 2018. Multiplex polymerase chain reaction detection of Shiga toxin genes and antibiotic sensitivity of Escherichia coli O157: H7 isolated from beef meat in Quetta, Pakistan. J. Food Saf. 38:e12540. https://doi .org/10.1111/jfs.12540.

Setterington, E. B., and E. C. Alocilja. 2011. Rapid electrochemical detection of polyaniline-labeled Escherichia coli O157: H7.
Biosens. Bioelectron. 26:2208-2214. https://doi.org/10.1016/j.bios 2010.09.036.

Shen, G., H. Xu, A. S. Gurung, Y. Yang, and G. Liu. 2013a. Lateral flow immunoassay with the signal enhanced by gold nanoparticle aggregates based on polyamidoamine dendrimer. Anal. Sci. 29:799-804. https://doi.org/10.2116/analsci.29.799.

Shen, G., S. Zhang, and X. Hu. 2013b. Signal enhancement in a lateral flow immunoassay based on dual gold nanoparticle conjugates. Clin. Biochem. 46:1734-1738. https://doi.org/10.1016/j .clinbiochem.2013.08.010.

Shi, L., L. Zhu, J. Guo, L. Zhang, Y. Shi, Y. Zhang, K. Hou, Y. Zheng, Y. Zhu, J. Lv, S. Liu, and Z. Tang. 2017. Self-Assembly of chiral gold clusters into crystalline nanocubes of exceptional optical activity. Angew. Chem. Int. Ed. Engl. 56:15397-15401. https://doi .org/10.1002/anie.201709827.

Sperandio, V., J. L. Mellies, W. Nguyen, S. Shin, and J. B. Kaper. 1999. Quorum sensing controls expression of the type III secretion gene transcription and protein secretion in enterohemorrhagic and enteropathogenic Escherichia coli. Proc. Natl. Acad. Sci. USA 96:15196-15201. https://doi.org/10.1073/pnas.96.26.15196.

Sugikawa, K., T. Kadota, K. Yasuhara, and A. Ikeda. 2016. Anisotropic self-assembly of citrate-coated gold nanoparticles on fluidic liposomes. Angew. Chem. Int. Ed. Engl. 55:4059-4063. https://doi .org/10.1002/anie.201511785.

Sun, J., J. Ji, Y. Sun, M. H. Abdalhai, Y. Zhang, and X. Sun. 2015. DNA biosensor-based on fluorescence detection of E. coli O157: H7 by $\mathrm{Au} @ \mathrm{Ag}$ nanorods. Biosens. Bioelectron. 70:239-245. https:/ /doi.org/10.1016/j.bios.2015.03.009.

Takalkar, S., H. Xu, J. Chen, K. Baryeh, W. Qiu, J. X. Zhao, and G. Liu. 2016. Gold nanoparticle coated silica nanorods for sensitive visual detection of microRNA on a lateral flow strip biosensor. Anal. Sci. 32:617-622. https://doi.org/10.2116/analsci.32.617.

Taranova, N. A., A. E. Urusov, E. G. Sadykhov, A. V. Zherdev, and B. B. Dzantiev. 2017. Bifunctional gold nanoparticles as an agglomeration-enhancing tool for highly sensitive lateral flow tests: a case study with procalcitonin. Microchim. Acta 184:4189-4195. https:/ /doi.org/10.1007/s00604-017-2355-4.

Tarr, P. I. 1995. Escherichia coli O157: H7: Clinical, diagnostic, and epidemiological aspects of human infection. Clin. Infect. Dis. 20:110. https://doi.org/10.1093/clinids/20.1.1.

Yang, Z., J. Song, Y. Dai, J. Chen, F. Wang, L. Lin, Y. Liu, F. Zhang, G. Yu, Z. Zhou, W. Fan, W. Huang, Q. Fan, and X. Chen. 2017. Self-assembly of semiconducting-plasmonic gold nanoparticles with enhanced optical property for photoacoustic imaging and photothermal therapy. Theranostics 7:2177-2185. https://doi.org/ 10.7150/thno.20545.

Zhang, L., Y. Huang, J. Wang, Y. Rong, W. Lai, J. Zhang, and T. Chen. 2015. Hierarchical flowerlike gold nanoparticles labeled immunochromatography test strip for highly sensitive detection of Escherichia coli O157: H7. Langmuir 31:5537-5544. https://doi .org/10.1021/acs.langmuir.5b00592.

Zhou, Y., L. Ding, Y. Wu, X. Huang, W. Lai, and Y. Xiong. 2019. Emerging strategies to develop sensitive AuNP-based ICTS nanosensors. Trends Analyt. Chem. 112:147-160. https://doi.org/10 .1016/j.trac.2019.01.006

Zhu, M., Y. Wang, Y. Deng, L. Yao, S. B. Adeloju, D. Pan, F. Xue, Y. Wu, L. Zheng, and W. Chen. 2014. Ultrasensitive detection of mercury with a novel one-step signal amplified lateral flow strip based on gold nanoparticle-labeled ssDNA recognition and enhancement probes. Biosens. Bioelectron. 61:14-20. https://doi.org/10.1016/j .bios.2014.04.049

\section{ORCIDS}

Xiaolin Huang () https://orcid.org/0000-0002-8908-0443 Yonghua Xiong ๑ https://orcid.org/0000-0001-5141-0841 